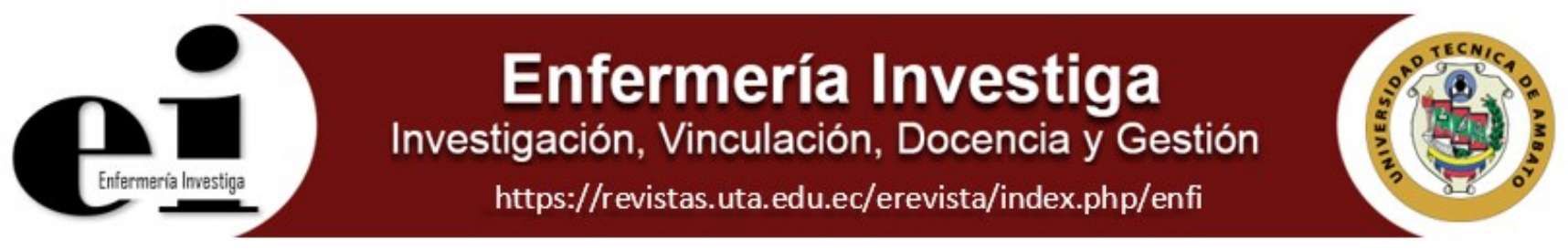

Artículo original

\title{
DETECCIÓN DE CHLAMYDIA TRACHOMATIS POR PCR EN TIEMPO REAL, EN RECIÉN NACIDOS EN EL HOSPITAL ISIDRO AYORA, QUITO-ECUADOR.
}

\section{DETECTION OF CHLAMYDIA TRACHOMATIS BY REAL-TIME PCR, IN NEWBORNS AT THE ISIDRO AYORA HOSPITAL, QUITO - ECUADOR.}

\section{Felicita, $\mathrm{M}^{1}$; Torres, $\mathrm{J}^{2}$; Achig, $\mathrm{M}^{3}$.}

${ }^{1}$ Lcda. Ana Maritza Felicita Mena, https://orcid.org/0000-0002-9444-8070

Laboratorio del Centro de Atención Temporal Quito Solidario (CAT)

${ }^{2}$ Dra. Nidian Judith Torres Noboa, https://orcid.org/0000-0002-0034-9988

Docente de la Universidad Central del Ecuador.

Laboratorio de Bacteriología y Virología. Facultad de Ciencias Médicas. Universidad Central del Ecuador.

${ }^{3}$ Ing. Mishell Cristina Achig Godoy, https://orcid.org/0000-0001-9649-1382

Laboratorio de Bacteriología y Virología. Facultad de Ciencias Médicas. Universidad Central del Ecuador.

Felicita, A; Torres, N; Achig M. DETECCIÓN DE CHLAMYDIA TRACHOMATIS POR PCR EN TIEMPO REAL, EN RECIÉN NACIDOS EN EL HOSPITAL ISIDRO AYORA, QUITO-ECUADOR. Enferm Inv. 2020;5(3):46-52

2477-9172 / 2550-6692 Derechos Reservados @ 2020 Universidad Técnica de Ambato, Carrera de Enfermería. Este es un artículo de acceso abierto distribuido bajo los términos de la Licencia Creative Commons, que permite uso ilimitado, distribución y reproducción en cualquier medio, siempre que la obra original es debidamente citada.

\section{Historia:}

Recibido: 05 abril 2020

Aceptado: 20 junio 2020

Palabras Claves: Chlamydia trachomatis, qPCR, sacos conjuntivales, recién nacidos.. Keywords: Chlamydia trachomatis, qPCR,

conjunctiva, newborns
Autor de correspondencia: Lcda. Ana Maritza Felicita Mena. Laboratorio del Centro de Atención Temporal Quito Solidario. E-mail: mary.felicita77@gmail.com

\section{Resumen}

Introducción: La transmisión vertical de Chlamydia trachomatis se presenta del $60 \%-70 \%$ en los hijos de madres infectadas y el riesgo de adquirir conjuntivitis por Chlamydia trachomatis en estos neonatos varía entre el $18 \%$ al $70 \%$. Objetivo: detectar la Chlamydia trachomatis por medio de qPCR (Reacción en Cadena de la Polimerasa por Tiempo Real) en los sacos conjuntivales en recién nacidos por parto normal. Métodos: Se realizó un estudio no experimental, transversal, analizando muestras procedentes de 178 niños nacidos vivos, en el Hospital Gíneco-obstétrico Isidro Ayora, se determinó la presencia de Chlamydia trachomatis en muestras de saco conjuntival, por medio de la reacción en cadena de la polimerasa los laboratorios de Biología Molecular de la Pontificia Universidad Católica del Ecuador. Resultados: la edad gestacional de los neonatos más frecuente es a término $75.29 \%$ que corresponde a 124 pacientes, resultados positivos para Chlamydia trachomatis por qPCR fueron 19 que corresponde al $10.7 \%$ y los resultados negativos 159 que corresponde al $89.3 \%$ de pacientes, el grupo de edad materna de los neonatos con resultados positivos para Chlamydia trachomatis por qPCR, más frecuente es el de 14 19 años con $31.5 \%$. Conclusiones: La conjuntivitis neonatal por Chlamydia trachomatis es una entidad de subdiagnósticada, que no responde al tratamiento con antibióticos tópicos habituales, donde la amplificación de ácidos nucleicos por medio de la qPCR ha sido un importante avance en el diagnóstico, debido a que la amplificación es extremadamente sensible (capaz de detectar una sola copia genómica) y altamente específica, inclusive en pacientes asintomáticos.

\section{Abstract}

Introduction: The vertical transmission of Chlamydia trachomatis occurs in $60 \%-70 \%$ in the children of infected mothers and the risk of contracting Chlamydia trachomatis conjunctivitis in these affected neonates between $18 \%$ and $70 \%$. Objective: to detect Chlamydia trachomatis by means of qPCR (Real Time Polymerase Chain Reaction) in the conjunctival sacs in newborns by normal delivery. Methods: A non-experimental, cross-sectional study was carried out, analyzing analyzed samples of 178 live-born children, at the Gyneco-obstetric Hospital Isidro Ayora, the presence of Chlamydia trachomatis in conjunctival sac samples was determined by means of the chain reaction of polymerase molecular biology laboratories of the Pontifical Catholic University of Ecuador. Results: the most frequent gestational age of the neonates is at term $75.29 \%$ corresponding to 124 patients, positive results for Chlamydia trachomatis by qPCR were 19 corresponding to $10.7 \%$ and negative results 159 corresponding to $89.3 \%$ of patients, the group of maternal age of neonates with positive results for Chlamydia trachomatis by qPCR, more frequent is that of 14-19 years with $31.5 \%$. Conclusions: Chlamydia trachomatis neonatal conjunctivitis is an underdiagnosed entity, which does not respond to treatment with usual topical antibiotics, where nucleic acid amplification by means of qPCR has been an important advance in diagnosis, due to extremely sensitive amplification. (capable of detecting a single genomic copy) and highly specific, including in asymptomatic patients 


\section{Introducción}

Chlamydia trachomatis es una bacteria Gram negativa aerobia, no móvil patógeno del humano, y parásito obligatorio intracelular de las células eucariotas, pertenece al Orden Chlamydial, Familia Chlamydiaceae, una característica distintiva de las Chlamydias es presentar un ciclo biológico diferente al de todas las bacterias. El ciclo inicia con el Cuerpo Elemental (CE) o partícula extracelular que es la parte infecciosa y metabólicamente inactiva, mide 0.2 a $0.4 \mu \mathrm{m}$, al cabo de 2 a 3 horas el CE se diferencia en Cuerpo Reticular (CR) o intracelular, metabólicamente activo, mide aproximadamente $1.0 \mu \mathrm{m}$. Chlamydia trachomatis, es el patógeno bacteriano que prevalece en las Infecciones de Transmisión Sexual (ITS) a nivel mundial, los datos reportados por el Center for Disease Control and Prevention (CDC) establecen que se producen 3 millones de infecciones nuevas cada año en Estados Unidos, los grupos de edades en que se registran mayor número de casos oscila entre 15 a 19 años. El costo de las infecciones Chlamydiales no tratadas y sus complicaciones está calculado en 2 millones de dólares solo en Estados Unidos (1).

Alrededor del $85 \%$ al $90 \%$ de los casos de infección por Chlamydia trachomatis tanto en la mujer como en el hombre son asintomáticas y pueden persistir por algunos meses o años. En la mujer los dos síntomas más comunes son la descarga mucopurulenta desde el cérvix y una hipertrofia ectópica cervical, además incluyen síndrome uretral agudo, uretritis, bartolinitis, cervicitis en el tracto genital superior (endometritis, salpingo-oforitis o enfermedad pélvica inflamatoria), perihepatitis (síndrome de Fitz-Hugh-Curtis) y reactivación de artritis $(2,3)$. La infección en la uretra y en el tracto genital inferior puede causar disuria, descarga vaginal anormal o sangrado post-coital, mientras que la infección del tracto genital superior (endometritis o salpingitis) pueden manifestarse con un sangrado uterino intermenstrual y un malestar pélvico $(2,3)$. En mujeres con infecciones Chlamydiales no tratadas pueden tener severas complicaciones reproductivas que incluyen infertilidad, embarazos ectópicos y dolor pélvico crónico. Durante el embarazo está asociada a partos prematuros, rotura prematura de membranas, recién nacidos de bajo peso, muerte neonatal y endometritis post-parto (2).

La transmisión vertical de Chlamydia trachomatis se presenta del $60 \%$ al $70 \%$ en los hijos de madres infectadas (3) y el riesgo de adquirir conjuntivitis por Chlamydia trachomatis en estos neonatos varía entre el $18 \%$ al $70 \%(2,4-6)$. Las ITS principalmente, la producida por Chlamydia trachomatis, puede contaminar y producir una infección al recién nacido al pasar por el canal del parto, infección ocular y/o respiratoria. Existen reportes que mencionan que entre el 22 al $50 \%$ de los niños nacidos de una madre infectada desarrollará conjuntivitis de inclusión y entre un 10 a $20 \%$ neumonía $(2,4,5,6)$.
La conjuntivitis neonatal (CN) por Chlamydia trachomatis es más prevalente de lo sospechado particularmente en ambientes socio-económicos desfavorecidos, es importante identificar la fuente de contagio e iniciar un tratamiento precoz para prevenir las cicatrices en los casos que pudieran cronificarse. La conjuntivitis por Chlamydia trachomatis es una entidad sub-diagnosticada, presenta un curso subclínico autolimitado y suele interpretarse como una conjuntivitis vírica $(1,2,7,8)$.

En el Ecuador no existen datos o estudios de prevalencia de conjuntivitis neonatales y menos aún de conjuntivitis producidas por Chlamydia trachomatis, esta al ser reportada como uno de los agentes causales más importantes de conjuntivitis neonatal en otros países, se lo debe considerar como patógeno importante de estudio en la población.

La conjuntivitis neonatal por Chlamydia trachomatis es una entidad de difícil diagnóstico en el Ecuador, que no responde al tratamiento con antibióticos tópicos de uso habitual y que causa importante morbilidad en el período neonatal y de lactancia, e incluso puede comprometer la función visual hasta llegar a la ceguera, daños que pueden ser prevenidos con una profilaxis adecuada. $(9,10)$

Por la dificultad del diagnóstico en nuestro medio compararemos con otros países como Colombia que presenta una prevalencia en recién nacidos del 33\%, Chile 8\%, EEUU 18.7\%, Europa 1.2\%, Perú 19.7\% y México del 2 al $8 \%(1-3,11-16)$.

En el Ecuador se tiene una referencia de prevalencia del $6 \%$ obtenida de análisis realizados en muestras cervicales de mujeres adolescentes embarazadas en el Hospital Gíneco-obstétrico Isidro Ayora en el Departamento de Madres Adolescentes $(11,12,17)$, al igual que la referencia presentada en un estudio realizado en la ciudad de Ibarra en el Hospital San Vicente de Paul con una prevalencia en embarazadas en tercer trimestre del $23.02 \%(13,14,18,19)$.

Similar a la prevalencia mundial, la incidencia de Chlamydia trachomatis es variable en el mundo, así por ejemplo: en Argentina se presenta una incidencia 1.6 al $12 \%$ comparada con la incidencia de Estados Unidos que va del 1.4 al 4.4\% (1-20). Tampoco en nuestro país se conoce la incidencia de esta enfermedad $(1,8,21)$.

La introducción de los métodos de biología molecular en los laboratorios de microbiología clínica supone un gran apoyo a la hora de obtener diagnósticos sensibles y específicos en el menor espacio de tiempo posible. Estos métodos no sustituyen, sino que complementan los ya usados métodos microbiológicos tradicionales $(3,4,22)$. El análisis integrado de todos ellos está llevando a resultados más fiables y eficaces. Entre todas las técnicas moleculares, la Reacción en Cadena de la Polimerasa (PCR) ha adquirido un gran valor diagnóstico permitiendo la detección de agentes 
etiológicos, de sus genotipos de virulencia y resistencia con gran sensibilidad y rapidez $(1,2,4,20-22)$. El desarrollo de las técnicas basadas en la amplificación de ácidos nucleicos ha sido un importante avance en el diagnóstico de la infección chlamydial. (23-28) Debido a que la amplificación es extremadamente sensible (capaz de detectar una sola copia genómica) y altamente específica, ofrece la posibilidad de ser aplicada a técnicas no invasivas, pudiéndose aplicar a poblaciones que por ser asintomáticas no sería posible abordar de otra manera.(29-34) El método de diagnóstico con mayor sensibilidad (88\%) y especificidad (100\%) es la Reacción en Cadena de la Polimerasa qPCR(35-41)., por esta razón, el objetivo de esta investigación es determinar la prevalencia de Chlamydia trachomatis a través de la técnica de qPCR en los fondos de sacos conjuntivales de los recién nacidos por parto normal en el Hospital Gíneco-obstétrico Isidro Ayora, en Quito Ecuador.

\section{Materiales y métodos}

Estudio no experimental, transversal, se analizaron muestras procedentes de sacos conjuntivales de 178 neonatos nacidos en el Hospital Gíneco Obstétrico Isidro Ayora, El cálculo se hizo con la siguiente fórmula de muestreo aleatorio simple - MAS (40).

$$
\text { Fórmula } n=\frac{N * p * q * Z^{2}}{(N-1) e^{2}+p * q * Z^{2}}
$$

\section{$\mathrm{n}$ : tamaño muestral}

$\mathrm{N}$ : tamaño poblacional o universo (11300)

p: proporción $(0,197)$

$q: 1-p(0,803)$

z: nivel de poder $(1,64)$

e: nivel de seguridad $(0,05)$

Los criterios de inclusión: recién nacidos vivos por parto vaginal en el Hospital Gíneco-obstétrico Isidro Ayora, sin recibir profilaxis ocular, se excluyeron los niños cuyas madres habían recibido tratamiento antibiótico con eritromicina, azitromicina o amoxicilina en el último mes de embarazo.

Detección de C. trachomatis mediante Reacción en Cadena de la Polimerasa:

Toma de muestra: En el momento del nacimiento, antes de que se le administre la profilaxis ocular al recién nacido, se tomará una muestra de los sacos conjuntivales inferiores de ambos ojos con un hisopo de dacrón estéril, el mismo que se colocará en un medio de transporte M4RT.

La muestra colocada en el medio de transporte adecuado, permanecerá en refrigeración hasta ser transportada al laboratorio de Biología Molecular de la
Pontificia Universidad Católica del Ecuador (PUCE) para su procesamiento.

Los datos se analizaron mediante estadística descriptiva con medidas de tendencia central, dispersión, tablas, gráficos, evaluación de porcentajes, porcentajes y se determinó tasa de prevalencia. Para la estadística se creó una base de datos en EXCEL, datos obtenidos de las hojas de inclusión en el estudio y de trabajo de qPCR.

Se utilizó los cebadores KL1 y KL2 para amplificar un fragmento de $24 \mathrm{pb}$ del plásmido críptico de la Chlamydia (KL1: 5' TCC GGA GCG AGT TAC GAA GA 3' y KL2: 5' AAT CAA TGC CCG GGA TTG GT 3'). Realizamos la $\mathrm{PCR}$ en un volumen de reacción de 25 $\mathrm{mL}$ y la mezcla contenía MgCl2 1,5 mM, Tris- $\mathrm{HCl} 10 \mathrm{mM}$ $(\mathrm{pH} 9), \mathrm{KCl} 50 \mathrm{mM}$, Tritón $\mathrm{X}-100$ al $1 \%$, desoxinucleótidos trifosfatos $200 \mathrm{mM}$, cebadores $1 \mathrm{mM}$, Taq DNA polimerasa $1 \mathrm{U}$ (Promega).

El programa de amplificación consistió en una desnaturalización a $94^{\circ} \mathrm{C}$ por $5 \mathrm{~min}$, seguido de 35 ciclos de amplificación. Cada ciclo incluyó un paso de desnaturalización a $94^{\circ} \mathrm{C}$ por 1 min, un paso de unión a los cebadores a $55^{\circ} \mathrm{C}$ por 1 min y un paso de elongación a $72{ }^{\circ} \mathrm{C}$ por $2 \mathrm{~min}$. El paso de elongación final fue de 5 $\min$.

\section{Consideraciones éticas:}

Esta investigación se realizó conforme la Declaración de Helsinki para la realización de investigaciones médicas en humanos, con el correspondiente consentimiento informado de participación, aprobado por el comité de ética y/o revisor institucional., Además toda la información fue manejada confidencialmente y su empleo fue exclusivamente estadístico. La identidad de los sujetos se consideró información confidencial. En los formularios de recolección de datos fue tomado el nombre de la madre como referencia para la entrega de resultados y la identificación del paciente dentro del estudio fue a través de un número único de participación. El número del expediente clínico fue registrado y no tabulado para facultar el retorno al expediente clínico original en caso necesario. Se determinó que solamente las personas involucradas en el estudio podrán tener acceso a la información (formularios, base de datos y otras documentaciones). Si por alguna regulación local o del comité de ética o de la autoridad hospitalaria se debiera permitir el acceso de terceras personas a esta información o a la documentación del estudio, se prestará atención a esta situación y se lo permitirá siempre y cuando las personas involucradas aseguren mantener la confidencialidad, correspondiente de la información proporcionada y firmen un documento al respecto. 
Autorización - Comité de Ética

Antes del inicio del estudio se presentó un protocolo de la investigación a la autoridad del Centro Hospitalario y al comité de ética o comité revisor institucional, según proceda. Únicamente con la autorización, aprobación o visto bueno del nivel correspondiente se procedió a la recolección de muestras e información.

\section{Resultados}

\begin{tabular}{lcrc}
\hline $\begin{array}{l}\text { Tabla N01.- Distribución según edad gestacional del } \\
\text { neonato }\end{array}$ & $\begin{array}{c}\text { Semanas } \\
\text { de } \\
\text { Gestación }\end{array}$ & $\begin{array}{r}\text { Frecuencia } \\
\left(N^{\circ}\right)\end{array}$ & $\begin{array}{c}\text { Porcentaje } \\
\%\end{array}$ \\
\hline Pretérmino & $<37$ & 33 & $18,53 \%$ \\
Término & $37-42$ & 124 & $75,29 \%$ \\
Postérmino & $>42$ & 11 & $6,18 \%$ \\
\hline Total & & 178 & $100,00 \%$ \\
\hline Fuente: Autores & & &
\end{tabular}

ANÁLISIS: En tabla $N^{0} 1$ se observa que la edad gestacional de los neonatos más frecuente es a término $75.29 \%$ que corresponde a 124 pacientes, seguido por el grupo pre-término con $18.53 \%$ correspondiente a 33 pacientes y el grupo pos-término con un $6.18 \%$ presenta 11 pacientes.

FIGURA No1 Neonatos con resultados positivos para Chlamydia trachomatis por qPCR

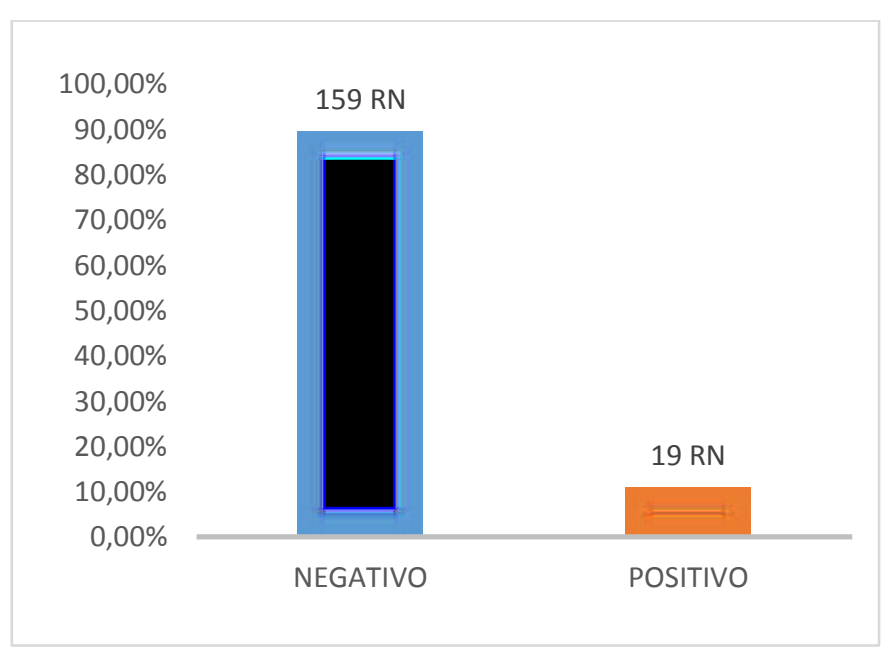

Fuente: autores
ANÁLISIS: En la figura №1 se observa que resultados positivos para Chlamydia trachomatis por qPCR fueron 19 que corresponde al $10.7 \%$ y los resultados negativos 159 que corresponde al $89.3 \%$ de pacientes.

\begin{tabular}{|c|c|c|c|}
\hline \multicolumn{4}{|c|}{$\begin{array}{c}\text { Tabla N02 Distribución de la edad Materna de Neonatos con } \\
\text { resultados positivos } \\
\text { para Chlamydia trachomatis por qPCR }\end{array}$} \\
\hline $\begin{array}{c}\text { EDAD EN } \\
\text { AÑOS }\end{array}$ & $\begin{array}{c}\text { Frecuen } \\
\text { cia de } \\
\text { edad } \\
\text { materna }\end{array}$ & $\begin{array}{l}\text { RN con } \\
\text { resultado } \\
\text { positivo por } \\
\text { qPCR (n19) }\end{array}$ & $\begin{array}{l}\text { Porcentaje de } \\
\text { positivos para } \\
\text { Chlamydia }\end{array}$ \\
\hline $14-19$ & 49 & 6 & $31.5 \%$ \\
\hline $20-24$ & 36 & 3 & $15.8 \%$ \\
\hline $25-29$ & 29 & 3 & $15.8 \%$ \\
\hline $30-34$ & 25 & 2 & $10.5 \%$ \\
\hline $35-39$ & 5 & 1 & $5.3 \%$ \\
\hline $40-44$ & 4 & 4 & $21.1 \%$ \\
\hline Total & 178 & $100.0 \%$ & $100.0 \%$ \\
\hline
\end{tabular}

Fuente: Autores

Se observa en tabla $\mathrm{N}^{\circ} 2$ que el grupo de edad materna de los neonatos con resultados positivos para Chlamydia trachomatis por qPCR, más frecuente es el de $14-19$ años con $31.5 \%$, que corresponde a 6 pacientes, seguido por el de 40 - 44 años con $21.1 \%$, que corresponde a 4 pacientes con resultados positivos para Chlamydia trachomatis por qPCR, en el grupo de 40 a 44 años de edad de la madre las 4 madres han contaminado a su hijos con Chlamydia trachomatis demostrándose se puede deber a una infección persistente y que permanece latente

\section{Tabla №3.- Distribución según edad gestacional.}

\begin{tabular}{ccc}
\hline Semanas de & Frecuencia & Porcentaje \\
\hline Gestación & $\mathbf{N}^{\circ}$ de Pctes & $\%$ \\
\hline 35 & 2 & $1,12 \%$ \\
36 & 3 & $5,61 \%$ \\
37 & 4 & $11,80 \%$ \\
38 & 5 & $17,42 \%$ \\
39 & 6 & $14,61 \%$ \\
40 & 7 & $30,34 \%$ \\
41 & 8 & $12,92 \%$ \\
42 & 9 & $3,93 \%$ \\
43 & 10 & $2,25 \%$ \\
\hline Total & 11 & $\mathbf{1 0 0} \%$ \\
\hline
\end{tabular}

Se observa en tabla $N^{\circ} 3$ que el grupo de edad gestacional más frecuente es el de 40 semanas con $30.3 \%(54)$, seguido por el de 38 semanas con $17.4 \%$ 
(31) una media de 39.13 semanas \pm 1.58 días con un rango de 35 a 43 semanas.

Tabla N.․ 4 Distribución según el peso del recién nacido.

\begin{tabular}{ccc}
\hline Peso & Frecuencia & Porcentaje \\
& $\mathbf{N}^{\circ}$ de Pctes & $\%$ \\
\hline $2000-2500$ & 18 & $10.11 \%$ \\
$2501-3000$ & 61 & $34.26 \%$ \\
$3001-3500$ & 78 & $43.82 \%$ \\
$3501-4000$ & 19 & $10.67 \%$ \\
$>4000$ & 2 & $1.12 \%$ \\
\hline Total & 178 & $100.0 \%$ \\
\hline
\end{tabular}

En la tabla $N^{\circ} 4$ el peso más frecuente es el del grupo de $3001-3500$ gramos (78) $43.82 \%$, seguido por el de $2501-3000$ (61) con $34,26 \%$ y con una media de 3187,22 gramos $\pm 1915,58$ gramos con un rango de 2060 a 4450 gramos.

En la figura $\mathrm{N}^{\circ} 2$ existe un $52.2 \%$ (93 RN) de sexo masculino y un $47,8 \%$ ( $85 \mathrm{RN}$ ) de sexo femenino lo que coincide con la distribución poblacional ecuatoriana.

FIGURA N² 2 Distribución según sexo del recién nacido

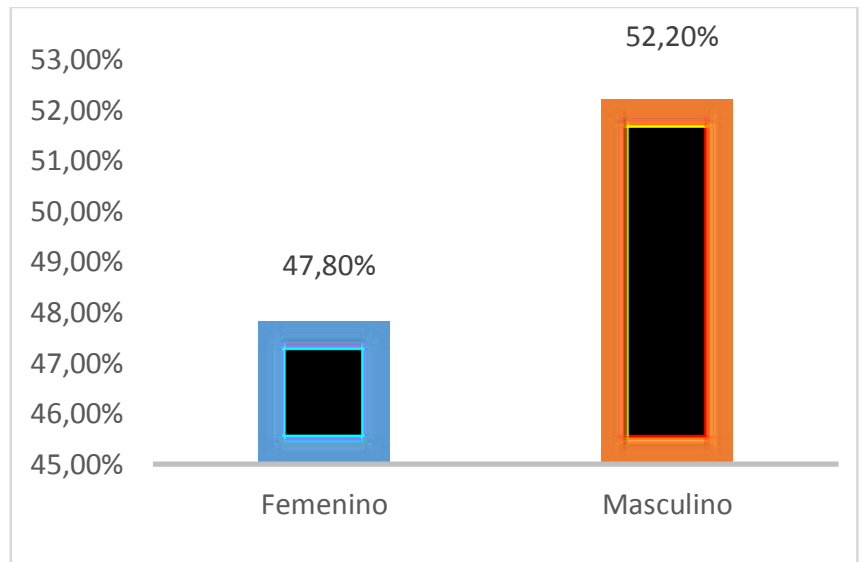

En la figura $\mathrm{N}^{\circ} 2$ existe un $52.2 \%$ (93 RN) de sexo masculino y un $47,8 \%$ ( $85 \mathrm{RN}$ ) de sexo femenino lo que coincide con la distribución poblacional ecuatoriana.

\section{Discusión}

Comparando con lo que se ha descrito en esta investigación, Colombia presenta una prevalencia del $33 \%$, Chile $8 \%$, Estados Unidos $18.7 \%$, Europa $1.2 \%$, Perú $19.7 \%$ y México del 2 al $8 \%$ todos estos datos fueron obtenidos de estudios realizados en sacos conjuntivales.

En Ecuador se tiene referencia de prevalencia del 6\% obtenida de análisis realizados en muestras cervicales de mujeres adolescentes embarazadas en el Hospital Gíneco Obstétrico Isidro Ayora en el Departamento de Madres Adolescentes, (Comunicación personal Dean, D. 2008), y en pacientes gestantes del área urbana de la ciudad de Ibarra, fue del $23.02 \%$ por qPCR (19).

En las últimas décadas Chlamydia trachomatis ha resultado ser el organismo prevalente entre los agentes causales de ITS con una prevalencia del 2 al $35 \%$ en embarazadas a nivel mundial la transmisión vertical está entre el $60 \%$ al $70 \%$ en los hijos de madres infectadas a nivel regional, tomando en cuenta los datos obtenidos en mujeres embarazadas ecuatorianas $(23.02 \%)$ y el resultado obtenido en esta tesis $(10.7 \%)$ en el Ecuador la transmisión vertical sería del $43.74 \%$.

La mayor prevalencia de Chlamydia trachomatis presentada por la CDC se observa en la adolescencia y en mujeres menores de 25 años por las características del epitelio endocervical, ya que el epitelio endocervical de las adolescentes es inmaduro y favorece la invasión de la Chlamydia trachomatis debido a que la unión escamo-columnar está oculta en la profundidad el ectocervix formando una zona central de epitelio columnar ectópico llamado ectropión, esta ectopia provee una amplia área de infección Chlamydial, en los resultados del presente estudio se aprecia que en el grupo de edad de 14 a 19 años indica la prevalencia $31.5 \%$ (6 pacientes) de resultados positivos para Chlamydia trachomatis por qPCR; Chlamydia trachomatis presenta infección latente y como corroboración de este dato tenemos que el grupo etario de 40 a 44 años presenta el $21.1 \%$ (4 pacientes) de positividad para Chlamydia trachomatis por qPCR.

El método de diagnóstico utilizado para el desarrollo de este estudio qPCR nos brinda el $88 \%$ de sensibilidad y $100 \%$ de especificidad comparado con métodos tradicionales, por ejemplo cultivo en células de Mac Coy $80 \%$ de sensibilidad y $100 \%$ de especificidad siendo este método el más cercano en sensibilidad y especificidad pero en nuestro medio es menos accesible que qPCR.

\section{Conclusiones}

- Los resultados encontrados en este estudio demuestran que la prevalencia es del $10.7 \%$ con una tasa de prevalencia del 4.06 por 1000 nacidos vivos, demostrando que Chlamydia trachomatis es una agente presente en nuestra población.

- $\quad$ Este estudio es una base para la determinación de prevalencia de Chlamydia trachomatis el mismo que puede ser utilizado para seguir determinando incidencia y realizar seguimiento alrededor de este tema.

- $\quad$ Chlamydia trachomatis es uno de los agentes causales de enfermedad de transmisión sexual con una prevalencia del $2 \%$ - $35 \%$ en mujeres embarazadas a 
nivel mundial, la transmisión vertical está entre el $60 \%$ al $70 \%$ en los hijos de madres infectadas y el riesgo de adquirir conjuntivitis por Chlamydia trachomatis en estos neonatos varía entre el $18 \%$ y $70 \%$. La prevalencia encontrada en este estudio es del $10.7 \%$ lo que nos coloca dentro del rango de las prevalencias a nivel mundial.

El diagnosticar Chlamydia trachomatis en recién nacidos vivos nos alerta de la circulación de este agente de transmisión sexual en nuestro medio; el Ecuador así presenta morbilidad sub diagnosticada que puede causar enfermedad en los productos como neumonía y conjuntivitis de inclusión que no son diagnosticados correctamente y mucho menos reportados.

\section{Recomendaciones}

- $\quad$ En vista de que la profilaxis ocular se realiza con nitrato de plata que es específico para Neisseria gonohorreae $y$ en ocasiones incluso solo con antibióticos tópicos como son los aminoglucósidos, que no cubren el espectro de acción sobre Chlamydias, se sugiere realizar una revisión al esquema de profilaxis ocular.

Se sugiere que en los controles pre-natales se investigue Chlamydia trachomatis para que el niño no se contamine y se pueda prevenir el desarrollo de conjuntivitis de inclusión.

- $\quad$ Es importante realizar un estudio que determine los agentes causales de conjuntivitis en el período neonatal en el Ecuador para que reciban un tratamiento específico.

- La educación continua individual y en grupo proporciona una forma de prevención de las diferentes complicaciones de esta patología, existe un desconocimiento de las enfermedades de transmisión sexual no clásicas por lo que se debe impartir más información de las enfermedades causadas por Chlamydia trachomatis, no son solo palabras o recomendaciones, confirmamos que se debe trabajar e insistir, no solo por un período corto sino a largo plazo y de manera continua.

La comunicación entre especialistas facilita un trabajo de calidad para sus pacientes involucrando una medicina preventiva.

Se recomienda promover las técnicas de biología molecular ya que estas tienen una especificidad del $100 \%$ y sensibilidad $88 \%$ que proporcionan diagnósticos certeros, a pesar que al momento los costos son elevados al ser solicitados con criterio clínico economizaría al paciente y se llegaría a un diagnóstico rápido y verídico.

Se sugiere evaluar a los pacientes que presentaron resultados positivos por qPCR para Chlamydia trachomatis de modo que se pueda determinar cuántos de ellos desarrollaron conjuntivitis y así obtener un valor real del riesgo de desarrollar conjuntivitis de inclusión en recién nacidos.
La infección por Chlamydia trachomatis es del tipo latente lo que quiere decir que permanece por meses o años en el tracto genital por lo que al nacer el niño por el canal del parto tiene riesgo de colonizarse y desarrollar conjuntivitis o pneumonia otros problemas son nacer con peso bajo, la recomendación es detectar a tiempo en los controles prenatales.

\section{Conflicto de intereses}

Ninguno declarado por los autores.

\section{Financiación}

Autofinanciado.

\section{Agradecimientos}

Ninguno declarado por los autores.

\section{Referencias}

1. Ostos OL, Sánchez RM. Chlamydia trachomatis: avances y perspectivas. Nova [Internet]. 2003;1(1):81-93. Disponible en: http://www.unicolmayor.edu.co/invest_nova/NOVA/artorevi1_1.pdf

2. Frontela Noda M, Amores S, Yepe Oliveros S, Kourí V, Ferreira Capote R, Mallea Sánchez L. Detección de Chlamydia trachomatis en muestras de exudado endocervical por la reacción en cadena de la polimerasa. Rev Cuba endocrinol. 2002;(August):135143.

3. Peipert JF. Genital Chlamydial Infections. N Engl J Med 2003;349(25):2424-30.

4. Vazquez LDP. Análisis epidemiológico de la infección por Chlamydia Trachomatis en Gipuzkoa (2006-2015): Prevalencia en gestantes, impacto en la población y genotipos circulantes. Universidad del País Vasco-Euskal Herriko Unibertsitatea; 2016.

5. López-Hurtado M, García-Romero S, Escobedo-Guerra MR, Bustos-López D, Guerra-Infante FM. Prevalencia de la infección genital por Chlamydia trachomatis en mujeres que asisten al Instituto Nacional de Perinatología de la Ciudad de México. Rev Chil infectología. 2018;35(4):371-6.

6. Villoslada J. Microorganismos asociados a infecciones cérvico-vaginales diagnosticadas por citología exfoliativa en el Hospital Regional Docente las Mercedes, Chiclayo 2017. 2018;

7. Di Bartolomeo S, Higa M, Janer M, Pennisi A, Balbin G, Priore G. Conjuntivitis neonatal en un hospital del Gran Buenos Aires. Situación de los últimos 5 años. Rev Argent Microbiol. 2005;37(3):139_ 41.

8. Millar K. Diagnóstico y tratamiento de la infección de la Chlamydia trachomatis. Am Fam Physician. 2006;78(8):1411-1416.

9. Cujilema C, Israel W. Etiopatogenia de conjuntivitis en recien nacidos. 2019;

10. Rivaya B, Lopez-Corbeto E, Lugo R, Matas L, González V, Casabona J. Pooling de muestras de orina para la detección molecular de Chlamydia trachomatis, Neisseria gonorrhoeae y Mycoplasma genitalium como estrategia de cribado entre la población joven en Cataluña. Enferm Infecc Microbiol Clin. 2020;38(2):65-71.

11. Quintero CA, Salafia C, Pennacchio GE, Dinamarca S. Diagnóstico molecular de Neisseria gonorrhoeae en Mendoza. Investig Cienc y Univ. 2019;3(4):36-42.

12. Carrera-Muiños S, Michel-Macías C, Fernández-Carrocera LA, Cordero-González G, Yllescas-Medrano E, Corral-Kassian E. Infecciones por gérmenes atípicos en el recién nacido hospitalizado en un centro de tercer nivel de atención. Un problema creciente. Perinatol y Reprod Humana. 2017;31(1):34-8.

13. Muñóz M, Romero F. P, Martínez T. A. Infecciones de transmisión sexual: Experiencia en una unidad de ginecología pediátrica. Rev Chil Pediatr. 2003;74(5):468-74. 
14. Zelaya L,Zelaya JL, Miranda U, Albites G, Zelaya A, Rojas G. Microorganismos presentes en cérvix uterino materno y sacos conjuntivales neontales. Rev Peru Enfermedades Infecc y Trop [Internet]. 2001;1:1-7. Disponible en: http://sisbib.unmsm.edu.pe/bvrevistas/speit/2001_n2/Microorganismo s_cervix_uterino.htm

15. Valencia C, Prado Valeria, Ríos M, Cruz M, Pilorget J. Trachomatis, Prevalencia de Chlamydia Determinada, en conjuntivitis neonatal Inmuno-, mediante las técnicas de Génica, fluorescencia y amplificación. Rev Med Chil [Internet]. 2000;128(7). Disponible en: https://scielo.conicyt.cl/scielo.php?script=sci_arttext\&pid=S0034-

98872000000700008

16. Rodriguez M d A, Correa E, Ospina Si, Giraldo C. Etiología bacteriana de la conjuntivitis neonatal. Colomb Med [Internet]. 1994;28(2):58-61. Available from: https://www.researchgate.net/publication/255993109_Etiologia bacte riana_de_la_conjuntivitis_neonatal_Metrosalud_Medellin_1994

17. Zapata M, Ahumada F, Cuffini C, Córdoba P, Grutadauria S. Aislamiento de Chlamydia trachomatis y respuesta inmune en diferentes poblaciones. Medicina (B Aires) [Internet]. 1997;57(1):7-14. Available

https://www.medicinabuenosaires.com/demo/revistas/vol57-

97/1/chlamydiatrachomatis.htm

18. Gómez LO, Bazante-Ramírez V. Prevalencia de Chlamydia trachomatis, Neisseria gonorrhoeae y Streptococcus agalactiae en mujeres embarazadas del área urbana de la ciudad de Ibarra-Ecuador. Rev la Fac Ciencias Médicas. 2012;37(1-2):10-7.

19. Ortiz L, Bazante M. Prevalencia de Chlamydia trachomatis, Neisseria gonorrhoeae y Streptococcus agalactiae, en pacientes gestantes del área urbana de la ciudad de Ibarra, año 2008. QUITO/PUCE/2010; 2010.

20. Curry SJ, Krist AH, Owens DK, Barry MJ, Caughey AB Davidson $\mathrm{KW}$, et al. Ocular prophylaxis for gonococcal ophthalmia neonatorum: US Preventive Services Task Force reaffirmation recommendation statement. Jama. 2019;321(4):394-398.

21. Bowling B,Kanski J. Oftalmología clínica. 8va ed. Elsevier, editor. Madrid; 2016. 63-65 p.

22. Mosmann J, Lopéz ML, Entrocassi AC, Cuffini C Prevalencia y análisis filogenético de Chlamydia trachomatis en una población de mujeres de Posadas, Misiones. Rev Esp Quim 2018;31(1):21-26.

23. Rodriguez J, Prado D. Microbiología: lo esencial y lo práctico. In: Organización Panamericana de la Salud [Internet]. 2005. p. 225-226. Disponible en: https://iris.paho.org/bitstream/handle/10665.2/51601/MicrobiologiaPra ctico_spa.pdf?sequence=1\&isAllowed=y

24. Irigoyen Coria A, Morales López H. Aspectos conceptuales de la ética de la investigación en medicina. Arch en Med Fam. 2017;15(2):31-5.

25. De Waaij D, Ouburg S, Dubbink J, Peters R, Morré S Evaluation of Prestoplus assay and LightMix kit Trichomonas vaginalis assay for detection of Trichomonas vaginalis in dry vaginal swabs. $\mathrm{J}$ Microbiol Methods [Internet]. 2016;127:102-104. Disponible en: http://www.sciencedirect.com/science/article/pii/S0167701216301312

26. Guerra F, Flores S, Arteaga G, Zamora A, López M, Ortiz F. Factores de riesgo y secuelas reproductivas asociados a la infección por Chlamydia trachomatis en mujeres infértiles. Salud Publica Mex. 2003;45(SUPPL. 5).

27. Rayo S, Peralta A, Baroja I. Frecuencia de Chlamydia trachomatis en mujeres de edad fértil al usar PCR en tiempo real en el
Servicio de Laboratorio del Hospital Carlos Andrade Marín. Rev Médica-Científica CAMbios HECAM. 2017;16(2):17-20.

28. Costa J. Reacción en cadena de la polimerasa (PCR) a tiempo real. Enferm Infecc Microbiol Clin. 2004;22(5):299-305.

29. Díaz Martín D, Úbeda Cantera M, López Suárez A, Álvarez de Mon Soto M. Respuesta inmune innata y sus implicaciones fisiopatológicas. Med [Internet]. 2017;12(24):1388-97. Available from: http://dx.doi.org/10.1016/j.med.2016.12.009

30. Jones J, Frausto RF, Langley S, Keefe KS, Aldave AJ, Affeldt J. Patología del epitelio corneal humano en el pénfigo vulgar mediada por anti-desmogleína 3. Rev Mex Oftalmol. 2019;93(4):169-77.

31. Hall GS. Bailey \& Scott's Diagnostic Microbiology, 13th Edn Lab Med [Internet]. 2013 Nov 1;44(4):e138-9. Disponible en:: https://doi.org/10.1309/LM5JCOPH0OGGBSZZ

32. Piñeiro L, Galán JC, Vall-Mayans M. Infecciones por Chlamydia trachomatis (incluye linfogranuloma venéreo) y Mycoplasma genitalium. Enferm Infecc Microbiol Clin [Internet]. 2019;37(8):525-34. Disponible en: http://www.sciencedirect.com/science/article/pii/S0213005X19301314

33. L. WCW(†)/ SDA/WMJ/EWK/ GWP/ PCS/ GLW. Koneman Diagnóstico microbiológico. Texto y Atlas en color. 6ta ed. S.A EMP, editor. Madrid; 2008. 1230-1234 p

34. Jawetz E, Melnick J, Adelberg E. Microbiología médica de. 27ed ed. Education M-H, editor. México, D.F; 2016:351 - 360.

35. Zucotti A, Bolaño L, Berruezo FA, Vitozzi S, Bottiglieri M Prevalencia de chlamydia trachomatis en embarazadas durante el primer trimestre en una institución privada de la ciudad de Córdoba. Rev Fac Cienc Med Cordoba. 2018;75(3):183-188.

36. Arias JEM, Franco KIG, Salazar DPP, Beltrán AEZ Conjuntivitis: revisión sistemática de diagnóstico y tratamiento. RECIAMUC. 2019;3(1):618-34.

37. Gazitúa FP, Araneda C, Araneda S, Solanes F, Seleme N, Ossandón D. Oftalmía Neonatal secundaria a Neisseria Gonorrhoeae: Reporte de un caso clínico y revisión de la literatura. Vis Pan-America, Pan-American J Ophthalmol. 2016;15(1):23-35.

38. Nogales MDC, Castro C, Ramírez M, Pueyo I, Pérez L, Jarana $R$, et al. Diagnóstico de la infección por Chlamydia trachomatis en un centro de diagnóstico y prevención de infecciones de transmisión sexual: Evaluación de los exudados cervicales, uretrales y rectales mediante técnica de PCR. Enferm Infecc Microbiol Clin. 2007;25(1):11-15.

39. Valderrábano LE. Alergia ocular en pacientes pediátricos. 2019.

40. Morales-Vallejo $P$. Tamaño necesario de la muestra: ¿Cuántos sujetos necesitamos? Estadística Apl a las Ciencias Soc [Internet]. 2012;24(1):22-39. Disponible en: https://s3.amazonaws.com/academia.edu.documents/50492311/lectu ra_42_la_importancia_del_tamano_de_muestra.pdf?responsecontent-disposition=inline $\% 3 \mathrm{~B}$

filename\%3DEstadistica aplicada a las Ciencias Soci.pdf\&X-Amz-

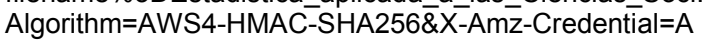

41. Persson K, Rönnerstam R, Svanberg L, Pohla MA. Neonatal chlamydial eye infection: An epidemiological and clinical study. $\mathrm{Br} J$ Ophthalmol. 1983;67(10):700-4. 